\title{
Room for dessert: an expanded anatomy of the stomach
}

$\mathrm{T}$ The presence of an accessory dessert pouch of the stomach has been postulated informally for years. These claims are often made near the end of a holiday feast, after the main course as thoughts turn to the pending arrival of pies, cakes, ice cream and pastries. A review of the medical literature, however, finds no mention of such a pouch. Indeed, the pouch has never been described in the anatomical record. Where, then, does dessert go, given that people often eat it after proclaiming themselves "full"? An alternative hypothesis suggests that dessert "fills in the cracks" between earlier courses. We developed the present study to address the hypothesis that an accessory pouch within, or attached to, the stomach provides the anatomic and physiologic requirements for dessert containment and absorption.

Radiographic examination of the stomach traditionally uses barium sulfate or Gastrografin as contrast agents. This study is exceptional because it pioneers the use of a novel contrast agent, chocoglucofructogalactomaltolactosucrografin (Fudge-o-grafin). Because the pouch is believed to hold only desserts, we hypothesize that a dessert-like contrast substance is required to demonstrate its presence.

\section{Methods}

Study subjects were found by contacting past winners and first runners-up of the Sturgeon Falls Blueberry Pie-Eating Contest $(n=30)$. The following exclusion criteria was established: fixed false belief that fruit salad is a dessert; history of "allergy" to chocolate; belief that cheese is acceptable as a dessert when not an ingredient of a cake; having already consumed dessert on the morning of the trials. All potential study subjects stated that they always have room for dessert. Five subjects were excluded due to their having already had a morning dessert. No history of deviant exclusionary dessert beliefs were elicited. The remaining 25 agreed to join the study on condition that they were provided with pie.

On the first day of the study a standard upper gastrointestinal series, using barium as the contrast agent, was performed on each subject. Next, after a week-long dessert-free fasting period, the same procedure was followed but the contrast agent used was Fudge-o-grafin. The radiographic plates were then studied by a team of radiologists, following double-blind procedures.

\section{Results}

A hollow pouch extruding from the stomach antrum was observed in all plates (IO०\%) made using Fudge-o-grafin, but in none $(0 \%)$ made using barium sulfate as the contrast agent. Eighteen of 25 subjects ( $72 \%$ ) complimented the flavour, smooth texture and pleasant aftertaste of the Fudge-o-grafin and I (4\%) requested more with which to wash down the post-test pie.

The pouch was found to vary only in size. In 5 cases (20\%) pouch volume was estimated to be $150-250 \mathrm{~mL}$. In 18 cases $(72 \%)$ the volume was estimated to be $250-500 \mathrm{~mL}$, while in the remaining 2 cases ( $8 \%$ ) the volume was estimated to be an exceptional I400-I600 mL. Each outpouching was shaped like a slice of pie, with the apex located at the antrum. The 
wall of the outpouching in each case was $5-6 \mathrm{~mm}$ in thickness, with rugae protruding into the lumen of the pouch at regular intervals of $8-9 \mathrm{~mm}$.

\section{Interpretation}

This radiographic study clearly demonstrates the presence of a previously undescribed anatomic phenomenon, to be known hereafter as the "Pot de Crème." The claim that dessert simply "fills in the cracks" has been absolutely refuted. In retrospect, the idea that "cracks" could exist between boluses of well-masticated food seems absurd.

This discovery is significant in and of itself. However, the questions raised by the presence of a pouch are perhaps even more important to our understanding of anatomy and physiology. First, is this pouch a genetic gift to a select few, or is it an endowment to all that undergoes atrophy in children without access to sufficient quantities of cookies, creams and chocolates? Second, Fudge-o-grafin may contain the key to an as yet undiscovered neurohormonal circuit, the details of which may be of commercial interest and are awaiting clarification.

\section{William D.J. Ruddock}

Department of Family Medicine

Queen's University

Kingston, Ont.

Sarah J. Kolk

Hespeler Clinic

Cambridge, Ont.

Angela J. Northey

Resident

Department of Anesthesia

Queen's University

Kingston, Ont.

and members of the MAPLE-SUGAR (Meat and Potatoes

Less Essential Than Sugar) Study Group

Conflicts of interest: The authors have interests in both chocolate and pie, and sometimes have conflicting thoughts about what to have for dessert.



